\title{
Applications of Spatial Light Modulators in Optical Signal Processing Systems
}

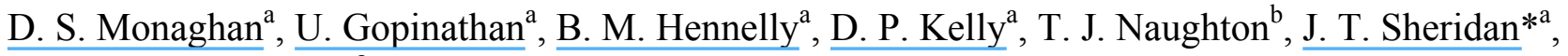 \\ ${ }^{a}$ Department of Electronic and Electrical Engineering, \\ University College Dublin, Belfield, Dublin 4, Republic of Ireland. \\ ${ }^{\mathrm{b}}$ Department of Computer Science, \\ National University of Ireland Maynooth, Maynooth, Republic of Ireland. \\ *Corresponding author: e-mail: John.Sheridan@ucd.ie \\ Tel: +353-(0)1-716-1927 Fax: +353-(0)1-283-0921
}

\begin{abstract}
Coherent optical signal processors, due to their ability to process and relay information in two dimensions, have been receiving increasing attention in recent years. These systems involve a coherent field being propagated through some bulk optical system consisting of thin lenses and sections of free space (such paraxial systems being described mathematically using the Linear Canonical Transformation). A Spatial Light Modulator (SLM) may be used to modulate the input digital data onto a coherent wave-field as well as to modulate the amplitude and/or phase of the complex wavefield at any desired plane. The complex field (amplitude and phase) at any desired plane may be recorded quantitatively using a CCD camera, using digital holographic techniques allowing the further processing of data digitally. Such hybrid optoelectronic systems have applications for 2D and 3D data processing covering fields as diverse as data storage, data security and pattern recognition. But devices such as SLMs and CCD cameras can represent only discrete levels of data necessitating a quantisation of continuous valued analog information. In this paper, we take the example of an optical system used to encrypt $2 \mathrm{D}$ and $3 \mathrm{D}$ data and evaluate the effect of the finite discrete levels of an SLM on the encryption/decryption process.
\end{abstract}

\section{INTRODUCTION}

Over the past few decades, Fourier Optics [1] has evolved into a mature and one of the most active research areas in Optics. Fourier Optics describes the theory of optical systems involving lenses separated by sections of free space in terms of Fourier and Fresnel transforms. There are two underlying assumptions (i) the Fresnel and (ii) the thin lens approximations. These approximations allow the description of the coherent field distribution between two planes separated by a free space distance in terms of a Fresnel transform and that between the front and back focal planes of a thin lens in terms of a Fourier transform. In general, optical field distribution at the input plane and output plane of any optical systems consisting of lenses separated by sections of free space can be described by a Linear Canonical Transform (LCT) [2]. The ease with which a Fourier transform can be realised using an optical system, undoubtedly one of the most important transform in signal processing, makes Optical Signal Processing [3,4] the single most important application of the theory of Fourier Optics. In fact, the terms Fourier Optics and Optical Signal Processing are almost synonymously used today. One of the main attractions of using optical systems for signal processing applications is that optical systems, being inherently two-dimensional, can process 2D information efficiently. When it comes to threedimensional information, holography is the most efficient way to record all information. Optical systems offer large space-bandwidth products which is very attractive for realising space-invariant operations. These operations are performed in the order of time it takes for an optical signal to propagate through the system at the speed of light. So, one is mainly limited by the delay and accuracy of the input and output optoelectronic devices. One of the most significant recent developments in the field of Optical Signal Processing have been made possible by the technological advances leading to development of high resolution CCD cameras and Spatial Light Modulators (SLMs). A CCD camera samples an optical field at regular intervals and outputs an electrical signal proportional to the intensity incident on each pixel. An SLM modulates a wavefront in both amplitude and phase depending on the input electrical signal. In other words, a CCD 
camera is an optical to electrical transducer and SLM is an electrical to optical transducer. This information can be now processed both in the optical and digital domains interchangeably. One of the most important issues here is the optimal sampling [5] of an optical field. There have been many studies on this topic [6]. Recently, Hennelly and Sheridan [7] addressed this problem by analysing the signal in the Wigner space. The Wigner distribution function (WDF) is a time (space) - frequency distribution which enables one to view the spatial and frequency distribution of a signal. The WDF of a one-dimensional signal has two dimensions and that of a two-dimensional signal has four dimensions. One could associate a space-bandwidth product (SBP) with a signal as well as an optical system [8]. The SBP of a signal and system is related to the area of WDF. When a signal propagates through an optical system, inorder for no loss of information the system WDF has to fully pass the signal WDF. If one keeps track of the WDF of a signal as it propagates through an optical system, one can determine the WDF and thereby the required SBP of the sampling device assuming sampling over a uniform grid (as most image sensors and sources do). Hennelly and Sheridan [7] have proposed a matrix method to analytically determine the WDF of a general N-dimensional signal. They have also proposed an efficient and fast algorithm [9] to calculate the discrete LCT which describes a general optical system consisting of thin lenses separated by sections of free space. When using the SLM as a wavefront modulator, ideally one would like to be able to achieve all complex values - all amplitude values in the interval $[0,1]$ and all phase values in the interval [0,2 $\pi]$ independently. But SLMs based on liquid crystals achieve only certain discrete amplitude and phase values in this interval. Some of the SLMs work in amplitude-mostly mode, i.e. they achieve a discrete set of amplitude values with little or no variation in phase, or they work in phase-mostly modes (e.g. Parallel aligned liquid crystal modulator) but most of the SLMs work in a coupled mode where there is a degree of coupling between the amplitude and phase. One of the approaches to achieve full complex modulation uses a cascade of two SLMs one working in the amplitude-mostly mode and the other working in the phase-mostly mode [10].

There have been three main approaches to represent complex valued data on SLM. The first is to map the values to be represented on to the values that an SLM can represent using a minimum Euclidean distance method [11]. The second approach referred to as Pseudo-random encoding [12], statistically approximates the desired complex values with those values that are achievable with a given SLM. Originally developed for phase-only modulators, pseudorandom encoding has been extended to modulators for which amplitude is a function of phase by transforming the phase statistics to compensate for the amplitude coupling. The third approach is to encode the complex valued data as a computer generated hologram $(\mathrm{CGH})[13]$ by using a group of pixels to represent a complex data. Compared to the CGH approach, the pseudorandom encoding method is a point oriented encoding method and uses the full available space-bandwidth product of an SLM. Complex-valued data, when represented on an SLM with a discrete number of levels leads to quantization errors. For a double random phase encoding system [14], Goudail et al. [15] and Javidi et al. [16] have studied how the coded image perturbations affect the decoded image. Naughton et al. [17, 18, 26] and Matoba et al. [19] have studied the quantisation effects in the context of compression of a complex-valued digital hologram. The other desirable feature (when using an SLM in an optical system especially for encryption and pattern recognition applications) is the large space-bandwidth product. For encryption applications where one uses an SLM to display the key to encrypt the data, a large space-bandwidth product of the SLM means that one can use a long key. On the other hand, a large space-bandwidth product would mean more pixels in a given active area, reducing the pixel size, which in turn makes the optical system less tolerant to misalignment errors of the SLM. Recently, Unnikrishnan et al. [20] have studied the effects of misalignment of SLM in a double random phase encoding system.

In this paper, we consider an optical system which encrypts both two-dimensional data. In addition to amplitude, optical systems offer many degrees of freedom to encode data such as phase [14, 20-23], polarization [24] and wavelength [25]. In our system, the encryption is done using a random phase mask in a Fresnel plane. The random mask which is the key to the encrypted data is displayed on an SLM. The large space bandwidth product of the SLM (number of pixels in the SLM) makes the size of the key large. The encrypted data captured by the CCD camera is complex valued white noise. When decrypting, the complex valued data is displayed on the first SLM and the decoding mask is displayed on the second SLM. We use a virtual optics method to simulate the encryption and decryption process. The Linear Canonical Transform algorithm proposed in [9] could simulate any such optical system. The display of the encrypted image and the decoding mask on the SLM results in quantisation depending on the number of discrete amplitude/phase levels offered by each SLM. We study the following three effects due to an SLM on the decoding process: (1) The quantisation of encrypted image,(2) The quantisation of the decoding phase mask, and (3) misalignment of the decoding phase mask. Through this study, we gain some insight into the limitations of the use of SLM in terms of accuracy, finite resolution and space-bandwidth product and misalignment tolerances in an optical system to encrypt/decrypt two-dimensional data. In Section 2, we describe the method for encryption and decryption. In Section 3, we discuss the virtual optical method to simulate the encryption/decryption process and study the quantisation and 
misalignment errors due to the SLM. In Section 4, we present some results from our simulation and finally we conclude with a short discussion of our results.

\section{ENCRYPTION AND DECRYPTION}

In this section, we describe the process of encryption and decryption of the data. Please refer to Fig.1. The following analysis is carried out in one-dimensional with extension to two dimensions straightforward. Let $\mathrm{f}(\mathrm{x})$ represent the input data to be encrypted. Let $\mathrm{L}_{\alpha 1, \beta 1, \gamma 1}$ and $\mathrm{L}_{\alpha 2, \beta 2, \gamma 2}$ represent LCTs corresponding to two combinations of lenses and free space propagation distances. The input signal $\mathrm{f}($.$) is transformed using \mathrm{L}_{\alpha 1, \beta 1, \gamma 1}$, multiplied by a random phase mask ' $\mathrm{R}$ ' and transformed again using $\mathrm{L}_{\alpha 2, \beta 2, \gamma 2}$.

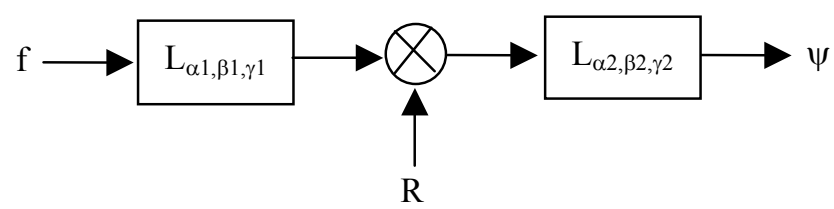

(a)

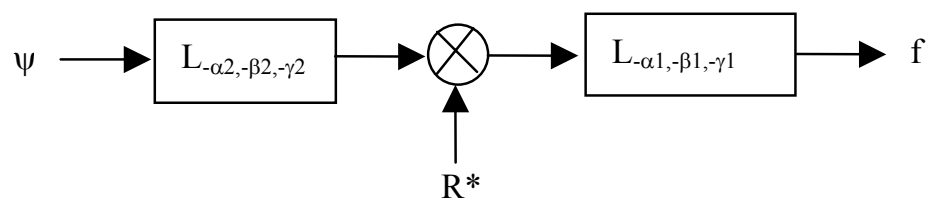

(b)

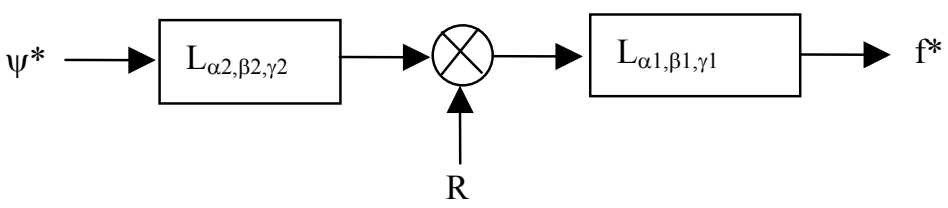

(c)

Fig. 1 Schematic of encryption and decryption method.

The LCT $\mathrm{L}_{\alpha, \beta, \gamma}$ of $\mathrm{f}($.$) is a three parameter transform described as$

$$
\mathrm{L}_{\alpha . \beta . \gamma}[f]\left(x^{\prime}\right)=K \iint f(x) \exp \left[j \pi\left(\alpha x^{2}-2 \beta x x^{\prime}+\gamma x^{\prime 2}\right) d x\right.
$$

The encrypted signal $\psi($.$) as in Fig. 1(a) can be written as$

$$
\psi(.)=\mathrm{L}_{\alpha 2, \beta 2, \gamma 2}\left\{\left\{\mathrm{~L}_{\alpha 1, \beta 1, \gamma 1}(\mathrm{f})\right\} \mathrm{R}\right\}
$$

As shown in Fig. 1(b) the encrypted signal could be decrypted in the following way:

$$
\mathrm{f}(.)=\mathrm{L}_{-\alpha 1,-\beta 1,-\gamma 1}\left\{\mathrm{R}^{*}\left\{\mathrm{~L}_{-\alpha 2,-\beta 2,-\gamma 2}\{\psi(.)\}\right\}\right\}
$$


where $\mathrm{L}_{-\alpha,-\beta, \gamma}$ is the inverse transform of $\mathrm{L}_{\alpha, \beta, \gamma}$ and ' $*$ ' indicates complex conjugate.

As shown in Fig. 1(c), there is a second method of decryption, suitable for optical implementation, using the following property of LCT

$$
L_{-\alpha,-\beta,-\gamma}\{f\}=\left[L_{\alpha, \beta, \gamma}\left\{f^{*}\right\}\right]^{*}
$$

Using the above property we can write eqn (3) as

$$
\mathrm{f}^{*}(.)=\mathrm{L}_{\alpha 1, \beta 1, \gamma 1}\left\{\mathrm{R}\left\{\left\{\mathrm{L}_{\alpha 2, \beta 2, \gamma 2}\left\{\psi^{*}(.)\right\}\right\}\right\}\right.
$$

If $\mathrm{f}($.$) is real, \mathrm{f}^{*}()=.\mathrm{f}($.$) . If one takes the conjugate of the encrypted signal and does the LCT operations used for$ encryption in reverse, one gets the conjugate of the input signal.

In the present work, $\mathrm{L}_{\alpha 1, \beta 1, \gamma 1}$ and $\mathrm{L}_{\alpha 2, \beta 2, \gamma 2}$ corresponds to free space distances ' $\mathrm{d}_{1}$ ' and ' $\mathrm{d}_{2}$ ' in which case

$$
\alpha 1=\beta 1=\gamma 1=\frac{\pi}{\lambda d_{1}} \quad \alpha 2=\beta 2=\gamma 2=\frac{\pi}{\lambda d_{2}}
$$

where ' $\lambda$ ' is the wavelength of light used.

\section{QUANTISATION AND MISALIGNMENT ERRORS DUE TO SLM}

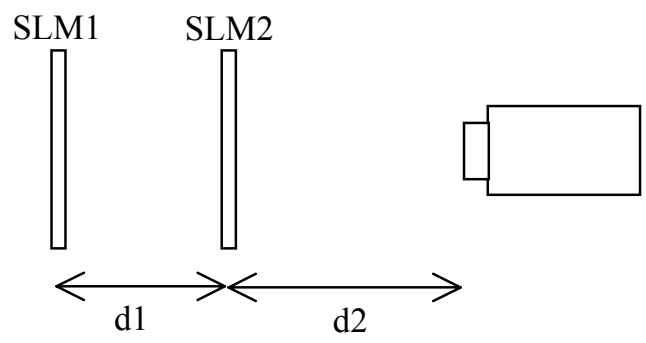

(a)

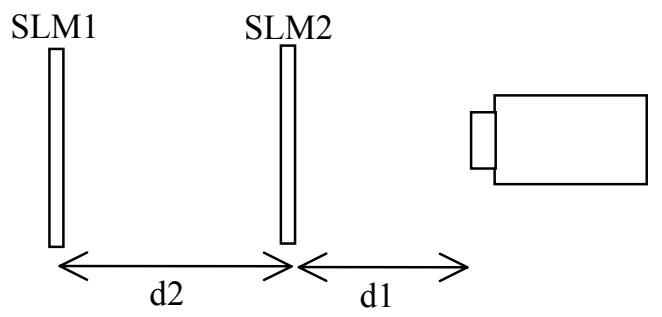

(b)

Fig. 2 Schematic of the optical system for encryption and decryption.

Fig. 2 shows the optical system which is used for encryption and decryption. In fig. 2(a), SLM1 and SLM2 are used to represent the input image and encrypting key respectively. In fig. 2(b), the SLM1 and SLM2 are used to display the encrypted image and the decryption key respectively. We perform the encryption and decryption process by a virtual optics method using the discrete Fresnel transform which is a special case of the discrete LCT and efficient algorithms for performing LCT in $n \log (\mathrm{n})$ time have been proposed recently [9]. The wavelength of the coherent source was 532 $\mathrm{nm}$, the distances $\mathrm{d} 1$ and $\mathrm{d} 2$ are $80 \mathrm{~mm}$, the CCD pixel size is 9 microns and the SLM pixel size is 36 micron. We assume a 100 percent fill factor for SLM and CCD.

The error in the decoded image when an SLM is used to represent the encrypted image is studied by modifying the encrypted image as follows:

$$
\psi_{\mathrm{i}}^{\prime}=\psi_{\mathrm{i}}+\Delta \psi_{\mathrm{i}}
$$

The where $\psi_{\mathrm{i}}$ represents the value of $\mathrm{i}^{\text {th }}$ pixel in the encrypted image, $\psi_{\mathrm{i}}^{\prime}$ represents the corresponding value represented by the SLM and $\Delta \psi_{i}$ is the resulting quantisation error in the $i^{\text {th }}$ pixel. If ' $a_{i}$ ' represents the amplitude part and ' $\phi_{i}$ ' represents the phase of the complex value $\psi^{\prime}$ i then

$$
\begin{aligned}
& \mathrm{a}_{\mathrm{i}} \in\{\mathrm{a} 1 \ldots . \mathrm{an}\} \text { for ' } \mathrm{n} \text { ' amplitude levels and } \\
& \phi_{\mathrm{i}} \in\{\phi 1 \ldots . . \phi \mathrm{n}\} \text { for ' } \mathrm{n} \text { ' phase levels }
\end{aligned}
$$


To study the error in the decoded image when an SLM is used to represent the decryption mask the decryption mask $\mathrm{R}^{\prime}$ is modified as follows:

$$
\mathrm{R}^{\prime}{ }_{\mathrm{i}}=\exp (\mathrm{i} \theta) \text { where } \theta \in\{\theta 1 \ldots \theta \mathrm{n}\} \text { for ' } \mathrm{n} \text { ' phase levels }
$$

The sensitivity of the position of the mask in the decryption process is studied by misaligning the position decryption mask by a fraction of a pixel in transverse ' $x$ ', ' $y$ ' and longitudinal ' $z$ ' directions.

$$
\mathrm{R}^{\prime}\left(\mathrm{x}^{\prime}\right)=\mathrm{R}(\mathrm{x}+\Delta \mathrm{x}, \mathrm{y}+\Delta \mathrm{y}, \mathrm{z}+\Delta \mathrm{z})
$$

where $\Delta \mathrm{x}, \Delta \mathrm{y}$, and $\Delta \mathrm{z}$ is the misalignment in the transverse ' $\mathrm{x}$ ', ' $\mathrm{y}$ ' and longitudinal ' $\mathrm{z}$ ' direction.

\section{RESULTS AND DISCUSSION}

In this section we present some results from our simulation study. Fig. 3(a) shows the 512 x512 grey level image used for our study. We corrupted the image by a random phase to make it correspond to a real world data corrupted by noise. The image is encrypted using the method shown in Fig. 1(a). The encryption mask has 256 discrete levels. First we study the quantisation effect of the SLM displaying the decoding phase mask. The phase of the decoding phase mask is quantised to different number of discrete levels. Fig. 3(b)-(d) shows the decrypted image quantised to 64, 8 and 2 levels. The corresponding plots for different number of levels are shown in Fig. 4. We use a metric normalised rms (NRMS) error in our study defined as

$$
\text { Normalised RMS error }=\frac{\sqrt{\sum_{i=1}^{N} \sum_{j=1}^{N}\left|I_{d}(i, j)-I(i, j)\right|^{2}}}{\sqrt{\sum_{i=1}^{N} \sum_{i=1}^{N}|I(i, j)|^{2}}}
$$

where $I_{d}($.$) and I($.$) are intensities of decrypted and original image respectively. In Fig. 4, it is seen that the error becomes$ significantly higher only if the number of discrete level is less than 10 .

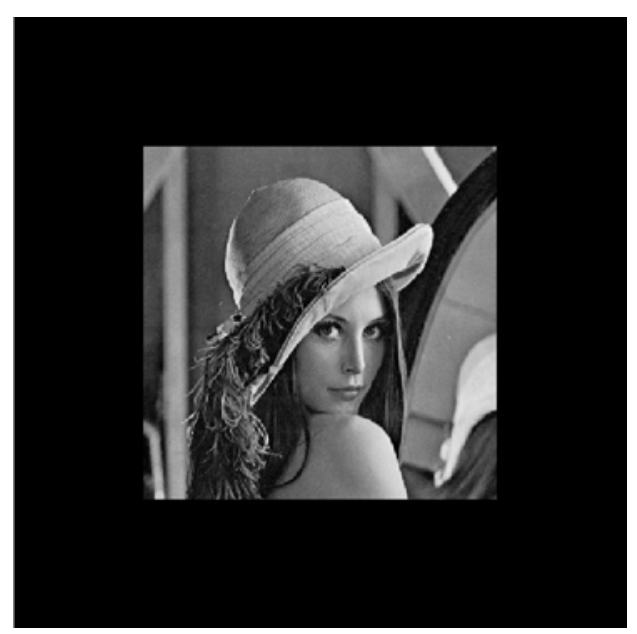

(a)

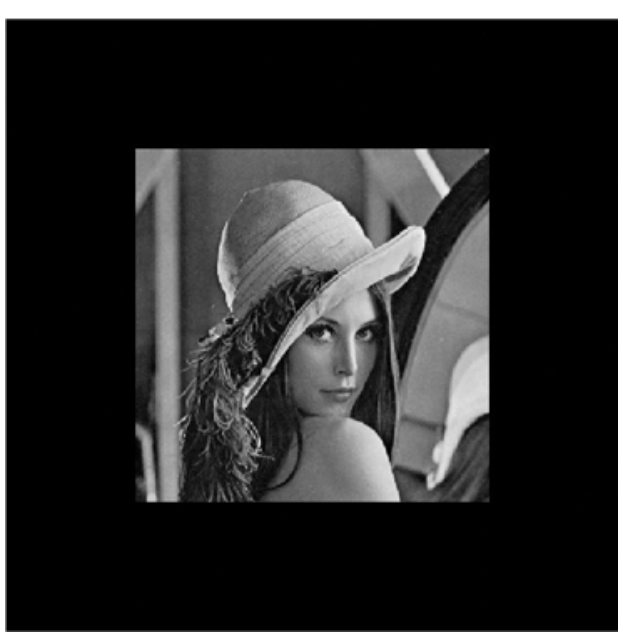

(b)

Fig. 3 


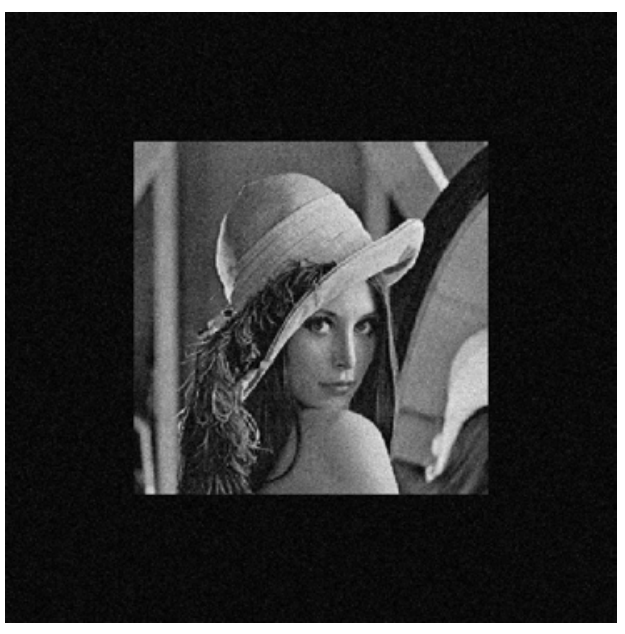

(c)

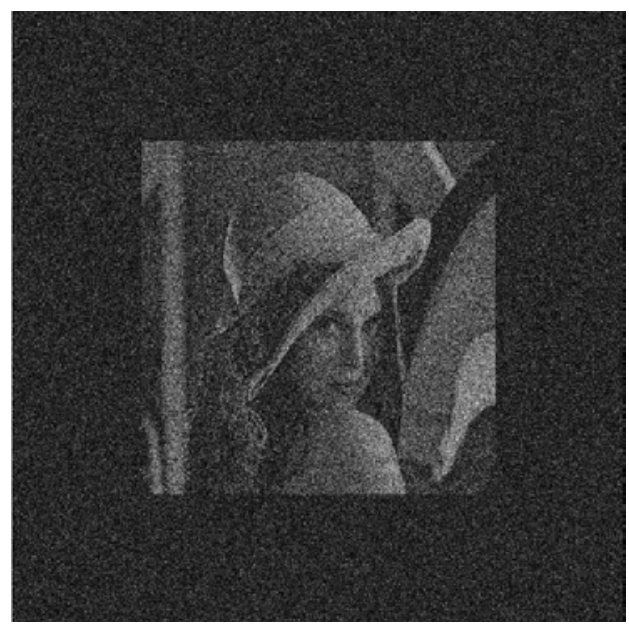

(d)

Fig. 3(a) the original image of size $512 \times 512$ (b), (c) and (d) the decrypted image with 64,8 and 2 quantisation levels of decoding phase mask respectively

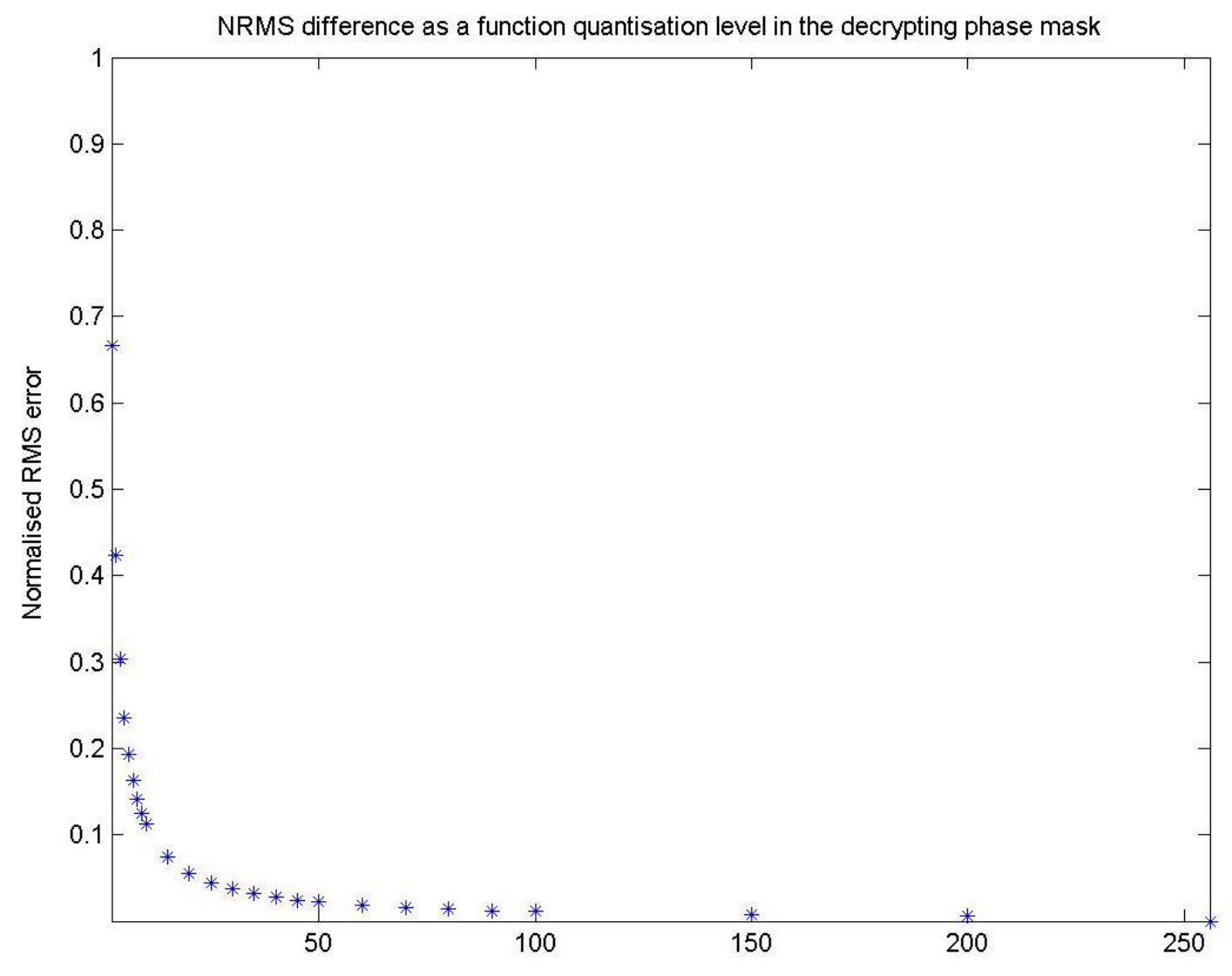

Number of levels of quantisation level in decrypting phase mask

Fig. 4 NRMS error between the decrypted image and the original image as a function of quantisation levels in the decrypting phase mask. 


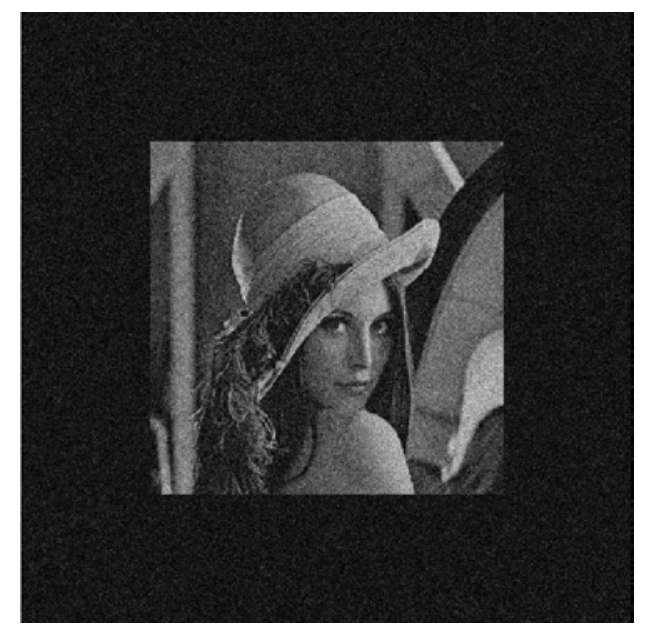

(a)

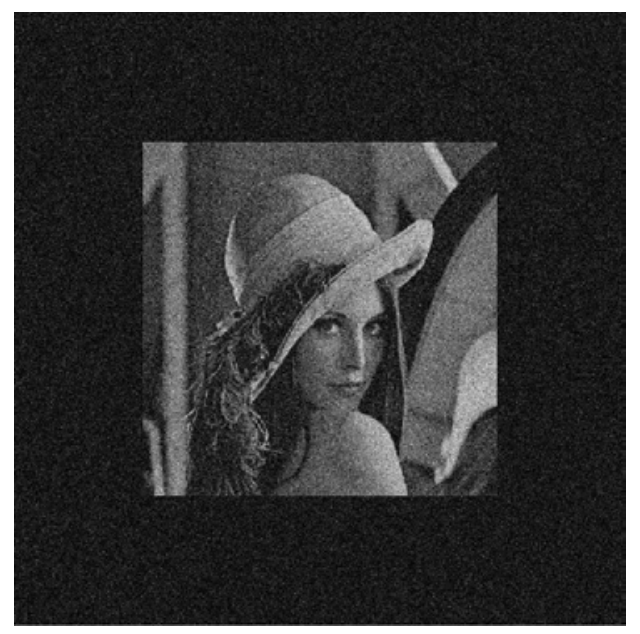

(b)

Fig. 5 (a) and (b) image decrypted using the phase of the encrypted image only quantised to 256 and 8 levels respectively.

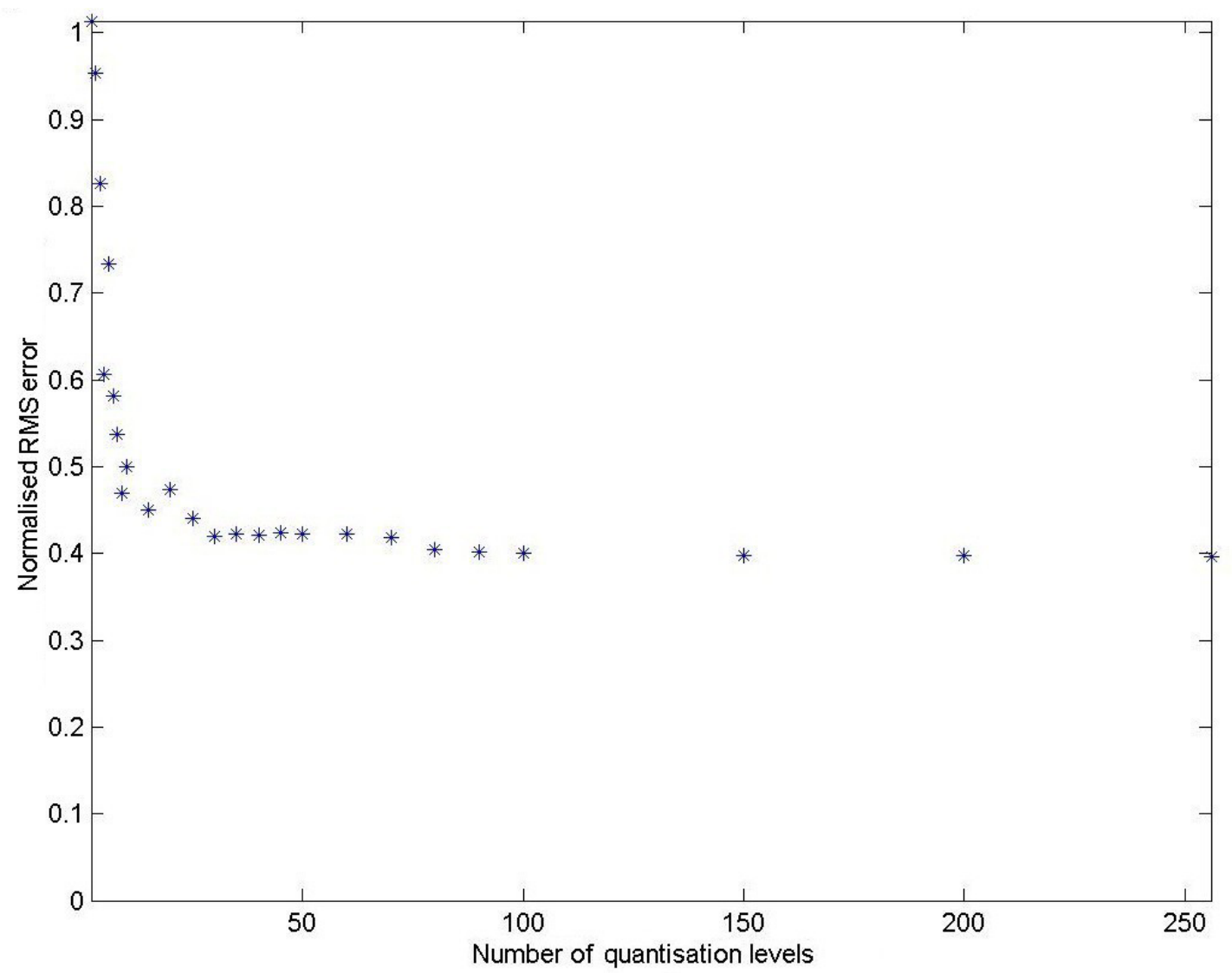

Fig. 6 NRMS error between the decrypted image and the original image as a function of quantisation levels in the encrypted image phase. 
The second study is the quantisation effect due to the finite discrete levels of the SLM displaying the encrypted image. We assume that the SLM is a phase-only SLM. We do not consider the amplitude of the encrypted image and consider only the phase. The phase of the encrypted image is quantised to different number of levels ranging from 2 to 256 . The resulting decrypted image is shown in Figs. 5 (a) and (b) corresponding to 256 and 8 levels. Fig. 6 shows the plot of the NRMS error for different number of levels ranging from 2 to 256. It may be noted that the error increases significantly when the number of quantised level falls below 10 .

Our third study is the errors due to the misalignment of the decoding phase mask in the $x, y$, and $z$ direction. Fig. 7(a) and (b) shows the misalignment in the x-direction, (c) and (d) in the y-direction and (e) and (f) in the z-direction. Fig. 8 shows the plot for different values ranging from -1 to $1 \mathrm{~mm}$. It may be noted from the plot that the decryption system is more sensitive to misalignment in the transverse (in plane) direction than in the longitudinal direction (along the optical axis).

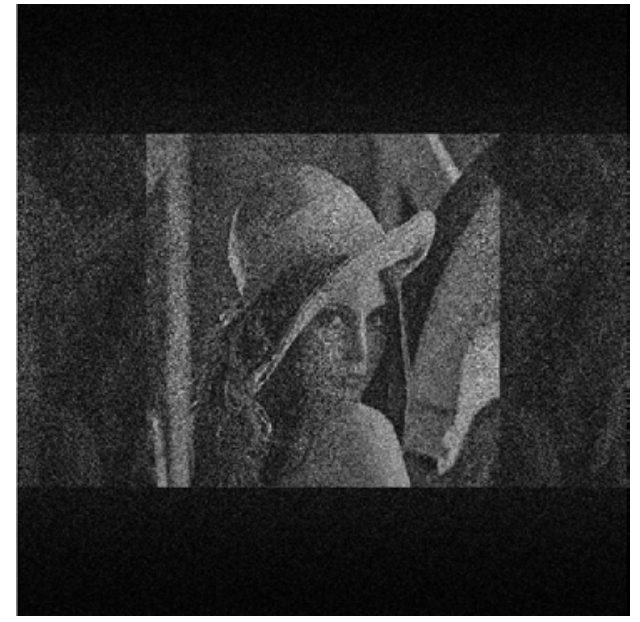

(a)

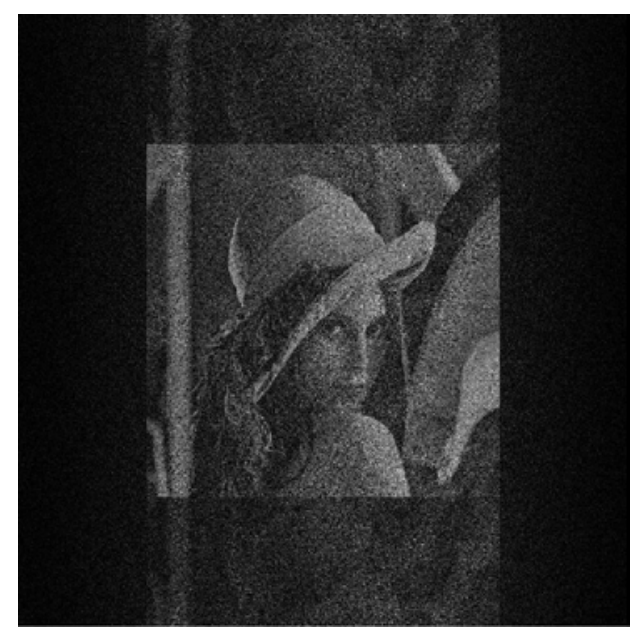

(c)

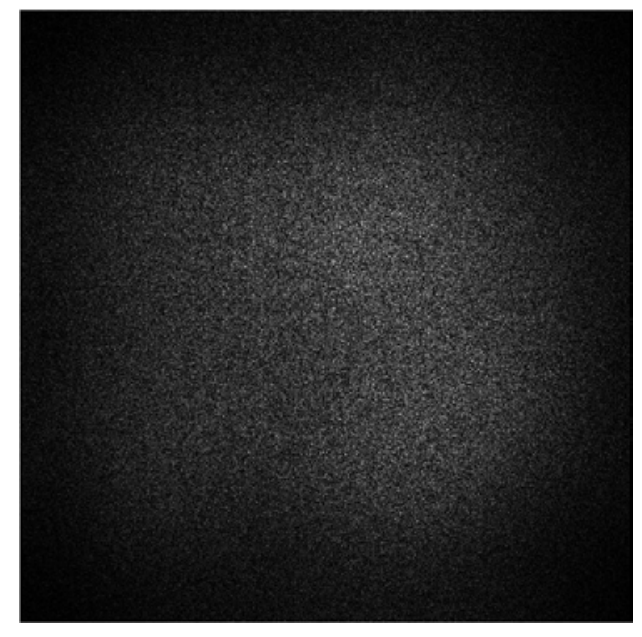

(b)

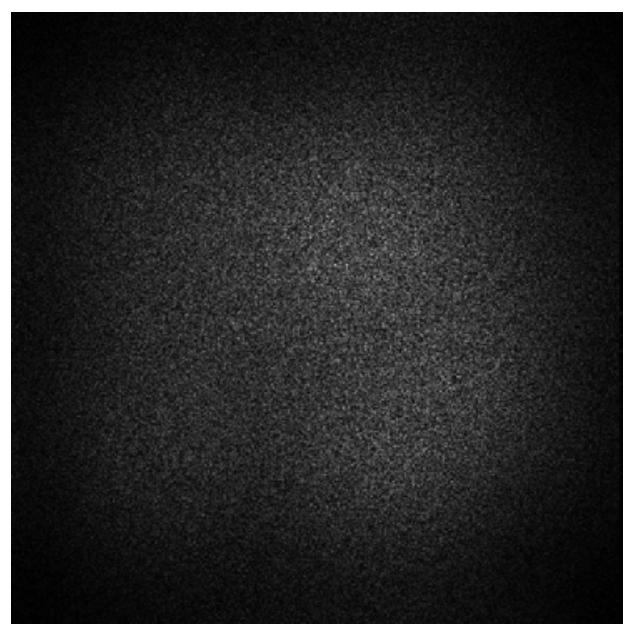

(d)

Fig. 7 


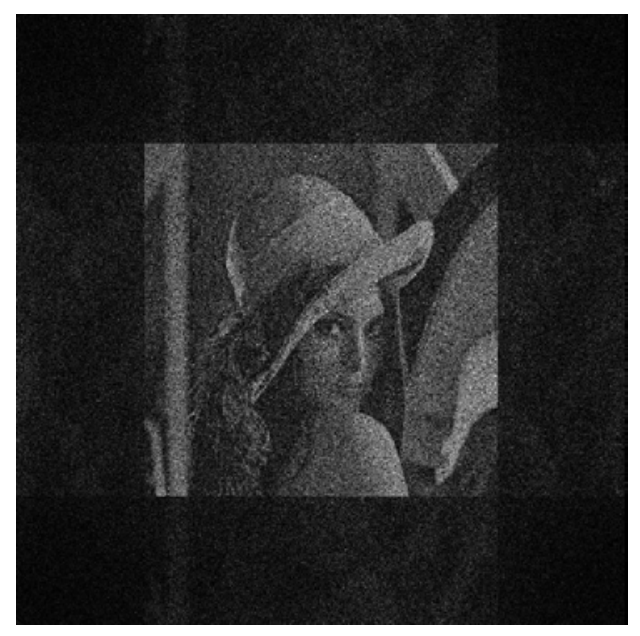

(e)

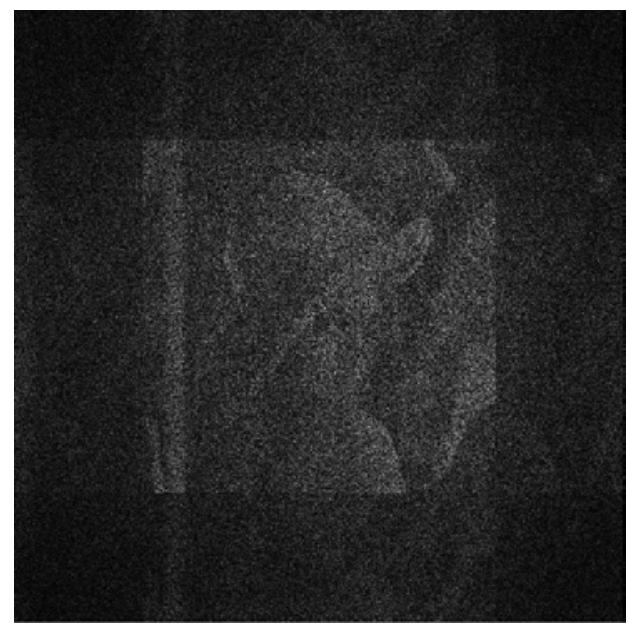

(f)

Fig. 7 (a) and (c) image decrypted with the decrypting phase out of alignment by $0.009 \mathrm{~mm}$ in the $\mathrm{x}$ and y axis respectively. (b) and (d) image decrypted with the decrypting phase out of alignment by $0.036 \mathrm{~mm}$ in the $\mathrm{x}$ and y axis respectively. (e) and (f) image decrypted

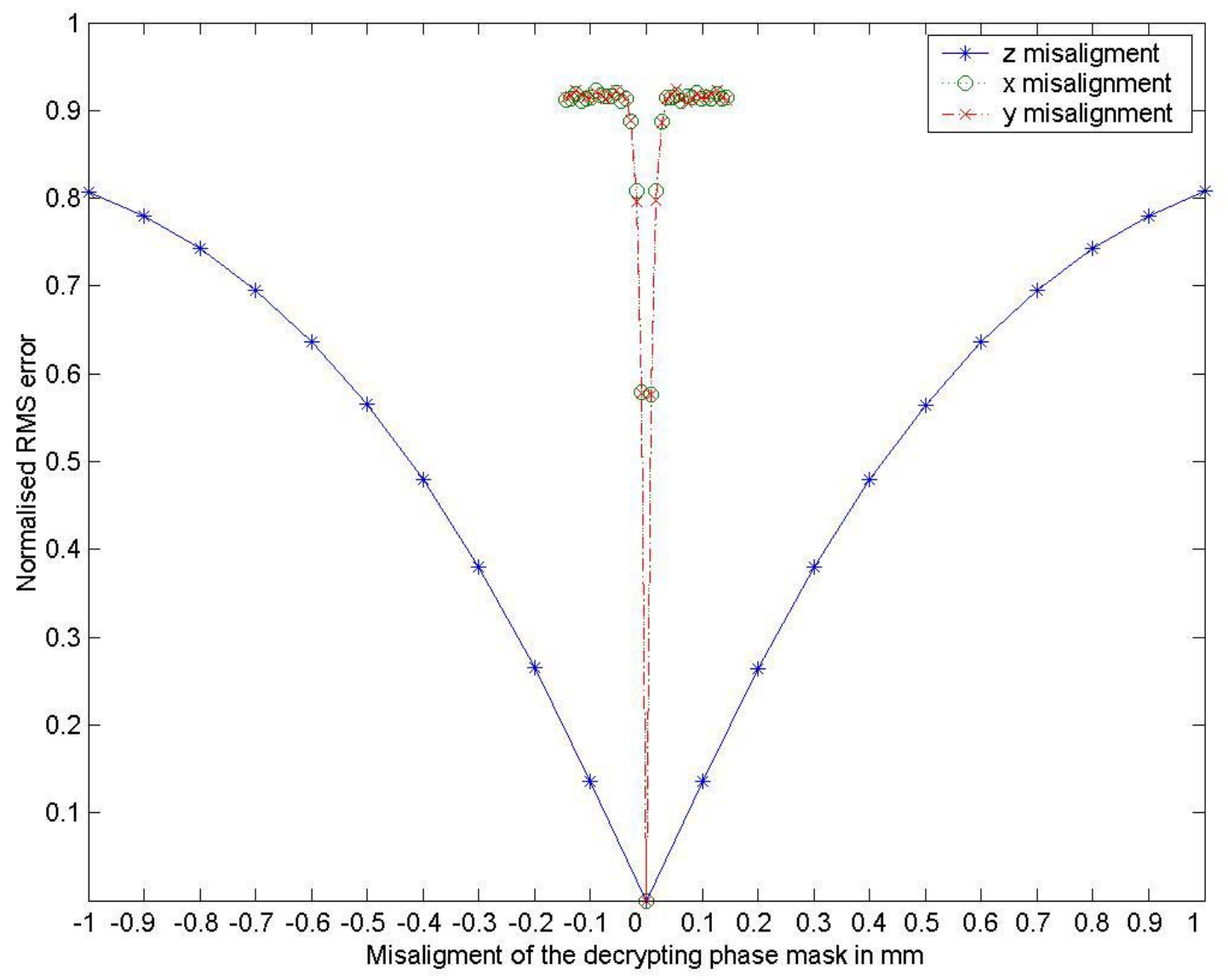

Fig. 8 NRMS error between the decrypted image and the original image as a function of misalignment of the decrypting phase for the $\mathrm{x}, \mathrm{y}$ and $\mathrm{z}$ axis. 


\section{CONCLUSION}

In this paper, we simulate the encryption and decryption of a two dimensional image. We study the sensitivity of the decoding system to the quantisation due to the finite number of discrete levels of the SLM used to represent the encrypted image and the decoding mask. The tolerance of the system to misalignment errors of the SLM used to represent the decoding mask is also studied. We use a phase-only SLM to represent the encrypted image and use only the phase information of the encrypted image for decryption. We note that the errors in decrypted image quantified using the NRMS parameter increase significantly only when the number of quantised levels fall below 10 . This shows the importance of phase information of the encrypted image. We also note from Fig. 6 that some information is fundamentally lost when the amplitude is equalised. Both of these issues have also been discussed by Matoba et al. [19]. We demonstrate that an SLM which can produce at least 10 discrete phase levels in the $[0,2 \pi]$ interval can be productively employed in optical systems to encrypt/decrypt images. We also find that the optical system is very sensitive to misalignment errors in the lateral ' $x$ ' and ' $y$ ' directions but a lot less sensitive to misalignment errors in the longitudinal direction along the axis of the optical system. The higher the space-bandwidth product of the SLM, the more sensitive is the optical system to misalignment errors of the SLM particularly in the transverse direction to the optical axis of the system.

\section{ACKNOWLEDGEMENTS}

We acknowledge the support of Enterprise Ireland and Science Foundation Ireland through the Research Innovation Fund and Basic Research Programme of the Irish Research Council for Science, Engineering and Technology.

\section{REFERENCES}

1. J. W. Goodman, Introduction to Fourier Optics, Third Ed.” Roberts \& Co. Publishers, Denver, 2004.

2. K. B. Wolf, Integral Transforms in Science and Engineering, Plenum, New York, Chap. 9 p. 381, 1979.

3. A. Vanderlugt, Optical Signal Processing, John Wiley \& Sons, 1992.

4. D. L. Flannery, J. L. Horner, "Fourier Optical Signal Processors", Proc. IEEE, Vol. 77, 1511-1527, 1989.

5. M. Unser, "Sampling-50 years after Shannon", Proc. IEEE ,Vol 88, 569-587, 2000.

6. A. Stern and B. Javidi, "Sampling in the light of Wigner distribution", JOSA A, Vol. 21, 360-366, 2004.

7. B. M. Hennelly and J. T. Sheridan, "Generalizing, optimizing, and inventing numerical algorithms for the fractional Fourier, Fresnel, and linear canonical transforms" JOSA A Vol. 22, May 2005 (to be published).

8. A. W. Lohmann, R. G. Dorsch, D. Mendlovic; Z. Zalevsky, C. Ferreira, "Space-bandwidth product of optical signals and systems" JOSA A, Vol. 13, 470-473, 1996.

9. B. M. Hennelly and J. T. Sheridan, "Fast numerical algorithm for the linear canonical transform", JOSA A Vol. 22, May 2005 (to be published).

10. L. G. Neto, D. Roberge, Y. Sheng, "Full-range, continuous, complex modulation by the use of two coupledmode liquid-crystal televisions", Appl. Opt., Volume 35, 4567-4576, 1996.

11. R. D. Juday, "Optimal realizable filters and the minimum Euclidean distance principle", Applied Optics, Vol. 32, 5100- 5111, 1993.

12. R. W. Cohn, "Pseudorandom encoding of complex-valued functions onto amplitude-coupled phase modulators", JOSA A, Vol. 15, 868-883, 1998.

13. P. M. Birch, R. Young, D. Budgett, C. Chatwin, "Two-pixel computer-generated hologram with a zero-twist nematic liquid-crystal spatial light modulator", Opt. Lett., Vol. 25, 1013-1015, 2000.

14. P. Refregier and B. Javidi, "Optical image encryption based on input plane and Fourier plane random encoding", Opt. Lett., Vol. 20, 767-769, 1995.

15. F. Goudail, F. Bollaro, B. Javidi, and P. Refregier, "Influence of a perturbation in a double phase-encoding system", JOSA A Vol. 15, 2629-2638, 1998.

16. B. Javidi, A. Sergent, G. Zhang, and L. Guibert, "Fault tolerance properties of a double phase encoding encryption technique", Opt. Eng. 36, 992-998 (1997). 
17. T. J. Naughton, Y. Frauel, B. Javidi, E. Tajahuerce, “Compression of Digital Holograms for Three-Dimensional Object Reconstruction and Recognition” Appl. Opt., Vol. 41, 4124-4132, 2002.

18. T. J. Naughton, J. B. McDonald, B. Javidi "Efficient Compression of Fresnel Fields for Internet Transmission of Three-Dimensional Images” Appl. Opt. Volume 42, 4758-4764, 2003.

19. O. Matoba, T. J. Naughton, Y. Frauel, N. Bertaux, B. Javidi, "Real-Time Three-Dimensional Object Reconstruction by Use of a Phase-Encoded Digital Hologram”, Appl. Opt., Vol. 41, 6187-6192, 2002.

20. G. Unnikrishnan, J. Joseph and K. Singh, "Optical Encryption System that Uses Phase Conjugation in a Photorefractive Crystal”, Appl. Opt., Vol. 37, 8181-8186, 1998.

21. G. Unnikrishnan, J. Joseph and K. Singh, "Optical encryption by double-random phase encoding in the fractional Fourier domain”, Opt. Lett., Vol. 25, 887-889, 2000.

22. B. M. Hennelly and J. T. Sheridan, "Optical image encryption by random shifting in fractional Fourier domains" Opt. Lett., Vol. 28, 269-271, 2003.

23. B. M. Hennelly, J. T. Sheridan, "Random Phase and Jigsaw Encryption in the Fresnel Domain", Opt. Eng., Vol. 43, 2233-2238, 2004.

24. G. Unnikrishnan, M. Pohit and K. Singh, "A polarization encoded optical encryption system using ferroelectric spatial light modulator”, Opt. Commun., Vol. 185, 25-31, 2000.

25. O. Matoba and B. Javidi, "Encrypted optical storage with wavelength-key and random phase codes", Appl. Opt., Vol 38, 6785-6790, 1999.

26. T. J. Naughton, B. Javidi, "Compression of encrypted three-dimensional objects using digital holography," Opt. Eng., Vol. 43, 2233-2238, 2004. 